\title{
An ethnographic exploration of postoperative pain experiences among Ghanaian surgical patients
}

\author{
Adejumo, Oluyinka and Aziato, Lydia.
}

\begin{abstract}
The experience of pain associated with surgery has been a challenge for health care professionals for many years, and culture is said to influence pain. Purpose: This study focused on patients' experiences of POP and factors that affect POP. Design:

The study employed qualitative ethnographic principles. Method: Data were collected through individual face-to-face interviews. Data were saturated after analysing data from 13 patients from two hospitals in Ghana. Findings: Themes that emerged were the subjective nature of pain, which described pain dimensions and communication; psycho-sociocultural factors, such as personal inclinations and sociocultural background; and health system factors, such as personnel attitudes and health financing. Implications for future research and practice: Health professionals need to understand the sociocultural effects of pain in order to give effective care. Conclusion: The study highlighted the need for patient education and the importance that health care professionals understand context-specific factors that influence POP management.
\end{abstract}

KEY WORDS: Postoperative pain; patients' experience; culture; pain management; and pain response. 


\section{Background}

Postoperative pain (POP) management is considered inadequate, although efforts have been made over the years to ensure effective management of POP globally (Hutchison, 2007; Svensson, Sjöström, \& Haljamäe, 2000). It has been reported in a quantitative survey in Ghana that $70 \%$ of postoperative patients experienced moderate to severe POP (Clegg-Lamptey \& Hodasi, 2005). Factors that influenced patients' experience of pain were not explored.

Pain response has been associated with the cultural background and socialisation of the individual, highlighting that response to a painful stimulus is not an innate characteristic but rather learned through socialisation from the individual's culture (Beyer \& Knott, 1998; Ogala-Echejoh \& Schofield, 2010). Hence the culture of a group of people largely influences their behavior as it involves their beliefs, knowledge, practices, values and everyday life activities (Callister, 2003; Leininger, 1997). It can therefore be inferred that the expression of behavioral signs (verbal and non-verbal) depends on the culture of the individual.

Culture therefore determines how pain is perceived, the meaning assigned to the pain, how or whether a person reports pain, and how the person acts or responds when in pain (Abu-Saad, Pool, \& Tulkens, 1994; Lovering, 2006; Ramer et al., 1999). For example, in Jordan, Abu-Saad (1984) found that girls are more emotional, sensitive and expressive than boys when it comes to behavioral expressions, and this could account for behavioral differences in pain expression among men and women reported in some studies (Fillingim, King, Ribeiro-Dasilva, Rahim-Williams, \& Riley Iii, 2009). Pain experiences among post- cardiac surgery women depended on what the women's expectations of pain were, and none of them wanted to complain about their painful experiences; most did not want to use pain medication or waited until their pain was unbearable before they did so. The participants needed individualised information about self-management of pain after discharge, and had difficulties remembering the information they had received. In a Kenyan study, Hastings (1995) realised that the acceptable outward expressions of pain experiences differ between tribes, some viewing stoicism as desirable and others engaging in ululation (wailing), weeping and singing to express emotions.

Based on the premise that the culture of a group affects their pain expression, this article reports on patients' pain experiences and factors that influence POP management in Ghana. This report was derived from a broader ethnographic study for a doctoral thesis that sought to develop clinical guidelines for the management of POP in Ghana.

Design

\section{Methodology}

The study employed a focused qualitative ethnographic approach to fully understand the factors that influenced patients' POP experience within the medicosociocultural context of Ghana. 
Sample. The study involved postoperative patients who underwent general surgical procedures. The sampling technique adopted for the study was purposive and participants were recruited two to eight days after surgery.

Setting. The study was conducted at a tertiary health facility and a regional hospital in Accra, Ghana.

Ethics. Ethical approval was obtained from the ethics review board at the University of the Western Cape, South Africa and the Ghana Health Service Ethics Review Committee. Appropriate informed consent was obtained (signature or thumbprint) and permission was obtained to record interviews.

Data collection method. Multiple data collection methods were employed in the parent study, such as individual interviews, clinical observation and clinical chart reviews. This article focuses on data generated through individual patient interviews. Interviews were conducted in English, Twi and Ewe as preferred by participants to enable ease of expression. Interviews lasted $30-45$ minutes and patients were not in pain at the time of the interviews. Individual interviews were conducted in an assigned private room on the ward to ensure privacy. Participants were assured of anonymity and confidentiality in this study. Saturation of data was obtained after recruitment of 13 postoperative patients ( 7 females and 6 males), when no new information was generated from participants and themes identified concurrently were fully developed. Identification codes used to represent participants are FP1 to FP7 for the female patients and MP1 to MP6 for the male patients.

Interviews were recorded with a digital voice editor which allowed transfer of recorded voice files onto a personal computer; the software allows direct transcription from the computer. Transcription was done by the first author and a trained research assistant, and the transcripts were identified with appropriate identification codes. The discussions conducted in Twi (the most common local dialect in the study setting) and Ewe were transcribed into English, focusing on the meaning of the participants' comments, and those conducted in English were transcribed verbatim (Esposito, 2001). To compensate for the omission of transcription in Twi and Ewe before translation, transcripts were reviewed by participants (member checks) and any vague or unclear comments were verified to ensure that the participants' views were accurately represented. Also, translations from Twi and Ewe were discussed with colleagues who were fluent in the languages, ensuring confidentiality and anonymity in the process. Direct Twi and Ewe transcription and back translation were omitted from this study due to budget constraints.

\section{Method of analysis}

The transcripts were read several times along with personal reflections, notes from patients' clinical documents and other documents on the ward to understand 
data generated. Hence data analysis occurred concurrently during the data collection to enable follow up on themes that emerged from the data. Identification of themes was carried out by reading through field notes, transcripts and personal reflections several times (Krueger, 1998; Rabiee, 2004). Reflexivity was ensured by keeping a detailed journal during data collection and analysis, where personal reflections and perceptions were written so that emic experiences did not bias the participants' accounts.

Data generated were analysed for thematic content analysis (Burnard, 1991). However, all the steps of Burnard (1991) were not followed during the analysis process because data were managed with NVivo 9, and Burnard's steps were formulated for manual data management. Thus steps for manual colouring of data and employing cutting and pasting of relevant areas of transcripts were not used in this study (Burnard's stages 8 and 9).

\section{Participant demographics}

\section{Findings}

The study involved male and female Ghanaian postoperative patients aged $18-50$ years who had undergone surgical procedures such as laparotomy, mastectomy, appendectomy, herniorrhaphy, haemorrhoidectomy and removal of a renal cyst, who shared their POP experiences. Participants were from various ethnic groups in Ghana, such as Akan, Ewe, Ga, Fante and Dagomba. Their occupations included teaching, nursing, trading, driving and baking, and some were housewives. They were all Christians. Four of the participants were illiterate, while the others were educated.

The study identified three main themes and corresponding sub-themes. The themes were the subjective nature of pain (sub-themes: dimensions of pain and pain communication), psycho-sociocultural factors (sub-themes: personal inclinations and sociocultural effect), and health system factors (sub-themes: personnel attitude and health financing). The findings are described and illustrated by participants' quotes.

\section{Subjective nature of pain}

Ghanaian postoperative patients' responses and descriptions of pain were strongly perceived to be individualistic and subjective. There was a clear indication that pain is a subjective phenomenon as all the participants described their personal pain experiences differently. The subjective nature of pain was succinctly described by MP3: 'as for pain, it depends on what is happening to you.' The dimensions of pain and various ways in which pain was communicated were explored and described. 
Dimensions of pain. Participants described pain intensity, quality or nature, and location of the pain. The intensity of pain was described as 'severe', 'much', and 'terrible.' For example, FP3 said 'initially, the pain was severe.' The patients also reiterated that the pain decreased with administration of analgesics. FP4 recalled that 'after the surgery, the severe pain I was experiencing had reduced ... the pain was on and off.' The intensity of pain described ranged from 'severe' to 'not much'. For example, MP1 said 'the pain was not much.'

The participants who reported that POP was 'not much' had experienced severe pre-operative pain ( $\mathrm{FP}_{1}, \mathrm{FP}_{3}, \mathrm{FP}_{4}, \mathrm{MP}_{1}, \mathrm{MP}_{3}, \mathrm{MP}_{4}, \mathrm{MP}_{5}$ and MP6). MP 3 , who underwent a laparotomy on account of intestinal obstruction, recounted as follows:

I was in severe pain before the surgery and I even cried ... but after the surgery, the pain was around the wound and even that was not as severe as the one I experienced before the operation.

Also, some participants (FP2, FP6, FP7, MP3 and MP5) described pain intensity as more severe in the night than during the day; $\mathrm{MP}_{5}$ : 'I was really in pain and I suffered that night till the next day.' Participants indicated that they 'suffered' when they were in severe pain. The quality or nature of pain felt after surgery was described as 'burning' and 'pulling' (FP2 and MP5). The location of pain was identified, such as 'abdomen', wound' and 'side of the wound'; for example, MP5 said 'the operated site was pulling me.' It was inferred that Ghanaian postoperative patients experienced a range of acute POP, from severe to slight pain. Participants described the pain immediately after surgery as 'severe', and as 'slight' when their condition improved. The invisible and personal nature of pain demanded that patients demonstrate its presence through both verbal and non-verbal behaviors.

Pain communication. Patients communicated or responded to pain through verbal and non-verbal behaviors or expressions. Pain behaviors expressed included 'shouting, crying, grimacing, and groaning'; for example, 'I was groaning but not too much' (FP5 and FP6). MP3 reported as follows: 'I was groaning; I was breathless; I grimaced when I was in pain; I couldn't sleep.'

It was noted that crying was not common among the postoperative patients. A few participants, both males and females, reported that they cried when the pain was very severe pre-operatively, but did not cry postoperatively. Male patient MP4 said: I frowned my face when I was in pain after the operation, I frowned my face; but I didn't cry ... the pain was not that severe for me to cry after the operation; but before I was brought to the hospital, I couldn't stand it, I couldn't do anything. I was in severe pain and I cried; I couldn't hide the pain.

Pain behaviors were noted to vary during the day and the night, with patients communicating that they had pain more often in the night. The patients reported 
that there were few nurses on night duty and the nurses were not available on the ward when they were in pain, so they had to shout to draw their attention. In the study setting two nurses routinely run the night shift, and this was considered inadequate by patients. Said MP2: 'I felt severe pain in the night and I was calling for help.' Also patients shouted for help at night perhaps because there was no bell or buzzers to call the nurses.

Patients also reported their pain to the health professionals such as nurses and doctors; they reported pain when the severity was high, both during the day and in the night. When patients reported pain some were given analgesics which helped reduce the pain and enhanced sleep. FP2 said: 'I told the nurses and they gave me some medicine, and after that the pain reduced.' Others were also reassured when they reported pain to the nurses. MP1 recalled as follows:

I called the nurses; actually when they came, the only thing they said to me was that 'it will go, it is a normal thing'.

Patients also reported some effects of pain after surgery such as: 'inability to 'sleep', 'walk', 'talk', 'turn in bed', and 'stretch in bed.' For example, FP2 said: 'I was walking slowly and slightly leaned forward'. Some of the patients believed that ambulation helped their recovery and pain management. For example, $\mathrm{FP}_{5}$ reiterated: 'I was feeling the pain but by the time you walk around, the pain is a little better.' There were individual differences during ambulation. For example, FP1 said: 'I was able to get out of bed the next day by myself; I didn't have much problems with the pain.'

\section{Psycho-sociocultural factors}

This theme describes factors that resulted from the patients' personal inclinations and sociocultural effects on pain response after surgery.

Personal inclinations. Pain response was influenced by decisions made prior to surgery and pain experiences before admission. Some patients did not verbally report their pain to health professionals due to their personal nature or inclination. For example, MP1 stated:

I just don't want people to know a lot about what is happening to me; so when I am feeling the pain I manage it myself. If there is any nurse around me then maybe I will inform her.

Sometimes such personal inclinations resulted from previous experiences which were related to previous pain experience and negative attitudes of nurses regarding pain management. Hence some patients experienced pain unnecessarily as a result of preconceived ideas. FP6, who had a previous surgical experience, recounted:

I had a previous caesarean section, so I knew how to handle myself. I know that if you call the nurses often, some of them become angry that you call too often, so when I am in pain, I try to endure it ... I try to control myself; I brace myself, so if they 
don't come to me, I don't call anybody unless they come to my bedside and I then tell them what is wrong with me.

Also, most patients had an 'expectation' of pain after surgery. Patients were 'content' that the diseased body part had been removed. Perhaps within the context of Ghana, postoperative patients accept some level of pain after surgery as 'normal'. The acceptable level of POP was not determined in this study as there was no pain assessment tool in use at the time of data collection. For example, FP4 said:

... there was pain; but I felt that whatever was in my stomach that was giving me the problem was no more; so I was not worried about the pain after the operation.

Sociocultural effect. Patients responded to pain as a result of the effect of social interactions and their cultural background. As patients interacted with each other and with relatives, they responded to pain based on the effect of such social interactions. For example, FP7 was advised by a relative not to 'talk' because the talking would increase the pain:

I couldn't talk, I was just lying there. I was so much in pain ... I was so quiet because I didn't want to talk for it to give me more pain ... I was also advised not to talk.

Some patients were able to bear pain because of their sociocultural background. Participants perceived that Ghanaians from the Northern part of Ghana were able to bear pain more than those from the South. This study was conducted in the Southern part of Ghana, but a few of the participants were from the North; FP7, a female patient from the Northern part of Ghana, corroborated this perception of participants:

... I was quiet; maybe that was how we were taught at home, like our mothers will normally tell us that if we cry, especially during childbearing, if someone is giving birth and she cries, they will tell you that you will remain like that in all your deliveries. So whenever you are going to give birth and if you cry, it means that you are spoilt or something, so normally when you are in pain you just hide it and it will go.

Again the social environment and upbringing of the patients influenced pain response. It was reported that a strict or difficult upbringing led to the ability to bear pain. MP1 said: 'I was trained in the military field, so even for you to see me crying, then you have to know that the thing is serious'.

Health system factors

This theme describes factors in the medical system of the study that had a bearing on response to pain among postoperative patients, such as health personnel attitude and health financing.

Personnel attitude. Patients' POP response was influenced by the attitude of health professionals such as nurses and doctors; positive and negative attitudes of health professionals affected patients' pain response. Positive attitudes encouraged reporting and expression of pain, and negative attitudes hindered patients' reporting 
pain. Patients referred mostly to the attitudes of nurses rather than doctors. For example:

... somebody is shouting and the nurse will run to the person and say 'Oh, because of this small thing that is why you are shouting?', then I better keep quiet because it means my pain is small if I am not shouting. (MP1)

Most patients were satisfied with the friendly communication by or relationship with health professionals (such as $\mathrm{FP}_{2}, \mathrm{FP}_{4}, \mathrm{FP}_{7}$ and $\mathrm{MP}_{3}$ ). The effect of such a friendly relationship on POP experience was not evaluated in this study. However, it was noted that the wider Ghanaian culture upholds friendliness and politeness among individuals, and this was echoed in this study. For example: They spoke to me politely and I was happy with the way they treated me' (FP4).

Amidst friendliness and the perceived 'good relationship', participants also experienced negative attitudes of nurses that resulted in undue POP. Also, patients perceived that a high workload prevented health professionals from giving adequate patient education. Therefore some Ghanaian postoperative patients did not have adequate knowledge of pain management:

... they didn't tell me what kind of drug, its effects or side-effects; I also don't know what drugs they give me. When they give me the drugs, I take it; I didn't ask them because I think they know the best drugs to give me. (MP5)

Health financing. The financial status of patients and their access to the National Health Insurance Scheme (NHIS) was related to availability of drugs such as analgesics. POP management was more effective if drugs prescribed were available.

Patients who were financially handicapped and did not have the NHIS were worried about how to pay their hospital bills on discharge, and this could have diverted their attention from any POP. At the time of data collection the NHIS covered the cost of some surgeries and drugs. Patients who were not registered wished they had the NHIS to defray the costs of their hospitalisation. Also, patients with NHIS who were admitted on weekends and holidays were not able to access the NHIS on the day of admission, because NHIS personnel do not work on those days. Financial deficit was regarded as a major concern for patients:

... at the moment, I am very worried about how my bill will be paid on discharge; ... I am only hoping that God will help me out. (FP3)

\section{Discussion and conclusions}

The findings of this study on issues such as the subjectivity of pain, dimensions of pain and factors that influence pain were to a large extent congruent with those of the wider literature. The phenomenon of pain construed as a subjective concept with a global connotation is supported by this study (McCaffery \& Pasero, 1999; Nielsen et al., 2008; Pasero, 2009). The individual differences of the POP experience require individualised pain management strategies to enhance pain management. The 
patients' reports of 'on and off', 'slight' and 'severe' pain also buttress the subjective nature of pain. The finding relates to the definition of pain, where the experiencing person is the one who can attest to the presence or absence of pain and the intensity of pain (McCaffery \& Beebe, 1989).

The description of pain and responses to pain within the medico-sociocultural context of this study draw attention to the individual nature of pain and the need for nurses to assess patients' pain after surgery. It was realised that some Ghanaian postoperative patients were reluctant to report their pain, and therefore there is a need to educate patients to report their pain to nurses as necessary. Also, it is reported that nurses within the context of the study identified their patients' pain through behavioral expressions exhibited by the patient rather asking about pain regularly (Aziato \& Adejumo, 2013a). The pain behaviors exhibited by patients in this study such as grimacing and moaning concur with those reported in the wider literature (Macintyre \& Ready, 2001; McCaffery \& Pasero, 1999), where variation of pain behavior among individuals has also been reported (Martin \& Todd, 2002; Murray et al., 2008).

The dimensions of quality and location described in this study are also important in the assessment and management of POP. For example, the location of pain may herald the development of complications, and early detection could save the patient's life. The effect of inadequately treated severe acute pain has been found to affect most systems of the body, such as the cardiovascular and respiratory system (MacLellan, 2006; Macintyre \& Ready, 2001). Some of the effects of inadequate pain management (for example in deep vein thrombosis) can have fatal consequences, and this makes effective POP management a priority care activity. Therefore the patients' reports of severe pain during the night, which supports previous findings (Closs, Briggs, \& Everitt, 1997; Closs, Gardiner, \& Briggs, 1998), should be a source of worry to health professionals. Effective POP management means that the patient's pain is relieved over the 24-hour period. It has been observed that during the night nurses find it more difficult to pick up non-verbal pain cues. Also, patients do not inform the nurses when they are in pain because they feel too tired and do not want to disturb the busy nurses. Thus patients rather prefer to wait until the nurses offer them analgesics (Carr, 1990; Closs et al., 1997).

The pain experienced among surgical patients can be related to the actual tissue damage that resulted from surgical incision, as supported by Brown, Christo and $\mathrm{Wu}$ (2004). However, in situations where patients are not willing to report their pain may defeat the global drive of POP management that desires patients to be painfree postoperatively (World Health Organization, 2007). The nurse should give patients opportunity to understand the importance of effective post-operative pain management so that they can actively participate in their pain management.

The sociocultural effects on pain experience demonstrated in this study were congruent with findings of previous studies in other countries, which confirm that 
pain response is affected by sociocultural context (Abu-Saad et al., 1994; Lovering, 2006; Ramer et al., 1999). The sociocultural background of the patient should be taken into consideration during the planning of pain management in order to enhance effectiveness. Also, patients should be encouraged to express their pain orientations developed from their sociocultural backgrounds, so that any misconceptions may be clarified. In this regard, it is necessary to adapt or develop a culturally appropriate pain assessment tool for pain assessment in Ghana. Insights gained from this study could inform the health team to adopt culturally specific methods of pain assessment and management. This study also identifies the need for further studies to validate such specific cultural methods for pain management. In order for the health team to communicate effectively with patients from different cultural backgrounds, the team should adopt cognitive and behavioral strategies that allow respect for the patients' culture.

Attitude of health personnel to POP management has been identified as a barrier to effective pain management; negative personnel attitudes hinder patients' reporting pain, as some do not want to incur the wrath of the nurses. Nurses have been found to contribute to ineffective management of POP. According to Coulling (2005), doctors and nurses made their own judgements about the patient's pain instead of relying on the patient's self-reporting thereof (McCaffery \& Pasero, 1999). Although patients reported a good relationship with health professionals, they still experienced unnecessary pain, and this substantiates the need for appropriate analgesic intervention for acute POP (Bell \& Duffy, 2009). Availability of analgesics was linked to the patients' access to health insurance and the economic status of patients and relatives. The surgical treatment option as an emergency process highlights the need for individuals to register for health insurance and also make other financial commitments towards their health care.

Implications for clinical practice. This study highlighted factors that warrant the need for postoperative patients within the sociocultural context of Ghana to be educated on effective pain control after surgery. This finding suggests that health professionals should bear the personal nature of pain in mind when managing pain, as patients respond to pain differently. The finding that some patients do not report their pain to nurses gives credence to previous research suggesting that POP management should follow regular administration of analgesics rather than giving drugs when patients request them (McCaffery \& Pasero, 1999).

Future research. It was realised that further investigation is warranted to confirm the perceived differences in pain response among Ghanaians from different cultural orientations.

Limitations of the study. The inability to carry out back translation of nonEnglish interviews was a deficit in this study. However, member checking was carried out to enhance true representation of participants' experiences. 
Conclusion. This ethnographic exploration allowed a full understanding of patients' postoperative pain experience. It was shown that some patients would not request analgesics on their own accord and suffered undue pain. Hence the study supports the need for timely and regular administration of analgesics among postoperative patients in Ghana.

\section{References}

Abu-Saad, H. H., Pool, H., \& Tulkens, B. (1994). Further validity testing of the Abu- Saad Paediatric Pain Assessment Tool. Journal of Advanced Nursing, 19, 1063-1071. Abu-Saad, H. H. (1984). Assessing children's response to pain. Pain, 19, 162-171.

Aziato, L., \& Adejumo, O. (2013). The Ghanaian Surgical Nurse and Postoperative Pain Management: A Clinical Ethnographic Insight. Pain Management Nursing doi: 10.1016/j.pmn.2012.10.002 (in-press).

Bell, L., \& Duffy, A. (2009). Pain assessment and management in surgical nursing: a literature review. British Journal of Nursing, 18 (3), 153-156.

Beyer, J., \& Knott, C. (1998). Construct validity estimation for the African-American and Hispanic versions of the Oucher Scale. Journal of Pediatric Nursing, 13, 20-31.

Brown, A. K., Christo, P. J., \& Wu, C. L. (2004). Strategies for postoperative pain management. Best Practice \& Research Clinical Anaesthesiology, 18 (4), 703-717.

Burnard, P. (1991). A method of analyzing interview transcripts in qualitative research. Nurse Education Today, 11, 461-466.

Callister, L. C. (2003). Cultural Influences on Pain Perceptions and Behaviors. Home Health Care Management \& Practice, 15 (3), 207-211.

Carr, E. C. J. (1990). Postoperative pain: patients' expectations and experiences. Journal of Advanced Nursing, 15, 89-100.

Clegg-Lamptey, J. N. A., \& Hodasi, W. M. (2005). An Audit of Aspects of Informed Consent and Pain Relief in General Surgical Units of Korle-Bu Teaching Hospital. Ghana Medical Journal, 39 (2), 63-67.

Closs, J. S., Briggs, M., \& Everitt, V. (1997). Night-time pain, sleep and anxiety in postoperative orthopaedic patients. Journal of Orthopaedic Nursing, 1, 59-66.

Closs, S. J., Gardiner, E., \& Briggs, M. (1998). Outcomes of a nursing intervention to improve post-operative pain control at night. Acute Pain, 1 (4), 22-31.

Coulling, S. (2005). Nurses' and doctors' knowledge of pain after surgery. Nursing Standard 19(34), 41-49.

Esposito, N. (2001). From meaning to meaning: The influence of translation techniques on non-English focus group research. Qualitative Health Research, 11(4), 568-579.

Fillingim, R. B., King, C. D., Ribeiro-Dasilva, M. C., Rahim-Williams, B., \& Riley Iii, J. L. (2009). Sex, Gender, and Pain: A Review of Recent Clinical and Experimental Findings. Journal of Pain, 10 (5), 447-485.

Hastings, F. (1995). Introduction of the use of structured pain assessment for post-operative patients in Kenya: Implementing change using a research-based co-operative approach. Journal of Clinical Nursing, 4, $169-176$.

Hutchison, R. W. (2007). Challenges in acute post-operative pain management. American Journal of Health-System Pharmacy, 64, S2. 
Krueger, R. (1998). Analyzing \& Reporting Focus Group Results: Focus Group Kit 6. London: Sage.

Leininger, M. (1997). Transcultural Nursing Research to Transform Nursing Education and practice: 40 Years. Journal of Nursing Scholarship, 29 (4), 341-348.

Lovering, S. (2006). Cultural Attitudes and Beliefs About Pain. Journal of Transcultural Nursing, 17 (4), 389- 395.

Macintyre, P. E., \& Ready, L. B. (2001). Acute pain management: A practical guide. London: W.B. Saunders.

Mac Lellan, K. (2006). Management of pain. United Kingdom: Nelson Thornes Ltd.

Martin, V., \& Todd, K. S. (2002). An evolutionary theory of pain must consider sex differences. Behavioral and Brain Sciences, 25 (4), 474.

McCaffery, M., \& Beebe, A. (1989). Pain: Clinical Manual for Nursing Practice. St Louis, USA: C.V. Mosby.

McCaffery, M., \& Pasero, C. (1999). Pain: Clinical Manual. London: Mosby Inc.

Murray, G., Judd, F., Jackson, H., Fraser, C., Komiti, A., Pattison, P., et al. (2008). Big boys don't cry: An investigation of stoicism and its mental health outcomes. Personality and Individual Differences, 44 (6), 1369-1381.

Nielsen, C. S., Stubhaug, A., Price, D. D., Vassend, O., Czajkowski, N., \& Harris, J. R. (2008). Individual differences in pain sensitivity: Genetic and environmental contributions. Pain, 136 (1-2), 21-29.

Ogala-Echejoh, S., \& Schofield, P. (2010). Systematic review on the literature on culture and pain. Journal of Pain Management, 3 (4), 347-354.

Pasero, C. (2009). Challenges in pain assessment. Journal of PeriAnesthesia Nursing, 24 (1), 50-54.

Rabiee, F. (2004). Focus-group interview and data analysis. Proceedings of the Nutrition Society, 63, 655-660.

Ramer, L., Richardson, J. L., Cohen, M. Z., Bedney, C., Danley, K. L., \& Judge, E. A. (1999). Multimeasure pain assessment in an ethnically diverse group of patients with cancer. Journal of Transcultural Nursing, 10 (2), 94-101.

Svensson, I., Sjöström, B., \& Haljamäe, H. (2000). Assessment of Pain Experiences after Elective Surgery. Journal of Pain and Symptom Management, 20 (3), 193-201.

World Health Organization (WHO). (2007). WHO Normative Guidelines on Pain Management: Report of a Delphi Study to determine the need for guidelines and to identify the number and topics of guidelines that should be developed by WHO. Geneva: WHO. 